\title{
Agreement Between Dual-Energy X-Ray Absorptiometry and Quantitative Ultrasound to Evaluate Bone Health in Adolescents: The PRO-BONE Study
}

\author{
Ana Torres-Costoso \\ University of Castilla-La Mancha \\ Dimitris Vlachopoulos \\ University of Exeter \\ Esther Ubago-Guisado \\ University of Exeter and University of Castilla-La Mancha \\ Asunción Ferri-Morales and Iván Cavero-Redondo \\ University of Castilla-La Mancha \\ Vicente Martínez-Vizcaino \\ University of Castilla-La Mancha and Autonomous University of Chile \\ Luis Gracia-Marco \\ University of Exeter, University of Zaragoza, and University of Granada
}

\begin{abstract}
Purpose: The present study aims to investigate the association between dual-energy X-ray absorptiometry (DXA) and quantitative ultrasound (QUS) parameters and the intermethods agreement in active males. Methods: In this cross-sectional study, bone health (by DXA and calcaneal QUS), physical activity (by accelerometers), and anthropometrics measurements were assessed in 117 active adolescents (12-14 y old). Bivariate correlation coefficients were calculated to assess the relationships between DXA standard regions of interest and QUS parameters. Intraclass correlation coefficients and Bland-Altman plots were used to assess the level of agreement between bone mineral content regions derived from DXA and stiffness index. The measurements were $z$ score transformed for comparison. Results: Most QUS parameters were positive and significantly correlated with DXA outcomes (stiffness index: $r=.43-.52$; broadband ultrasound attenuation: $r=.50-.58$; speed of sound: $r=.25-.27$ ) with the hip showing the highest correlations. Moreover, the present study found fair to good intraclass correlation coefficients of agreement (.60-.68) between DXA and QUS to assess bone health. The Bland-Altman analysis showed a limited percentage of outliers (3.2\%-8.6\%). Conclusion: QUS device could represent an acceptable alternative method to assess bone health in active adolescent males.
\end{abstract}

Keywords: stiffness index, bone mineral content, bone quality, calcaneal QUS

Osteoporosis is a skeletal disease characterized by decreased bone mass and abnormal bone microarchitecture, resulting in increased bone fragility (42). Bone mass acquisition during growth is an important modifiable determinant of skeletal health in adulthood $(6,24)$, with a peak bone mass achieved by the end of the second decade or very early in the third decade (2). Although the genetic predisposition largely determines bone mass, there are also environmental factors, such as physical activity (PA), that have been shown to predict bone mass during growth (34).

The investigation into skeletal growth related to PA may be important for the prevention of osteoporosis in later life. It is well known that certain sports induce osteogenic responses in the growing skeleton $(36,41)$. However, monitoring osseous changes over time is not an easy task. Dual-energy X-ray absorptiometry

Torres-Costoso and Ferri-Morales are with the School of Nursing and Physiotherapy, University of Castilla-La Mancha, Toledo, Spain. Vlachopoulos, Ubago-Guisado, and Gracia-Marco are with the Children's Health and Exercise Research Centre, Sport and Health Sciences, University of Exeter, Exeter, United Kingdom. Ubago-Guisado is also with the IGOID Research Group, University of Castilla-La Mancha, Toledo, Spain. Cavero-Redondo and Martínez-Vizcaino are with the Health and Social Research Center, University of Castilla-La Mancha, Cuenca, Spain. Martínez-Vizcaino is also with the Faculty of Health Sciences, Autonomous University of Chile, Talca, Chile. Gracia-Marco is also with the Growth, Exercise, Nutrition and Development Research Group, University of Zaragoza, Zaragoza, Spain; and PROFITH "PROmoting FITness and Health through physical activity" Research Group, Dept. of Physical Education and Sports, Faculty of Sport Sciences, University of Granada, Granada, Spain. Address author correspondence to Ana Torres-Costoso at AnaIsabel.Torres@uclm.es. 
(DXA) is currently the reference standard and the most widely used method to assess bone mineral content (BMC) and bone mineral density (BMD) in children and adolescents $(8,31,35)$, due to its time efficiency, precision, and the existing and robust pediatric reference data (5). However, it has some disadvantages, such as the 2-dimensional technique to predict $\mathrm{BMD}$, the ionizing radiation $(0.08-4.6 \mathrm{uSv})(19)$, and the fact that it is not portable, limiting its use to the laboratory environment. Therefore, large-scale and longitudinal studies might require techniques other than DXA that effectively estimate bone outcomes.

Quantitative ultrasound (QUS) measurement is considered a valid, safe, easy-to-use, portable, cost-effective, cheaper than DXA, and radiation-free method to assess bone health (1). The calcaneus site is the most frequent measurement site due to its trabecular content and accessibility (29). QUS includes the measurement of broadband ultrasound attenuation (BUA), speed of sound (SOS), and a mathematical combination of both BUA and SOS, defined as stiffness index (SI), which is the most widely used variable (11). In contrast to DXA, QUS parameters provide information about the quality of the bone, the elasticity, and the structural property of the tissues at the measured site (22). Previously, it has been reported to be a useful tool, similar to DXA, in assessing bone quality in elderly men and women $(16,21)$. In young adults, Wetter and Economos (40) reported that improved skeletal status assessed by QUS was associated with sports participation. Additionally, Yung et al (44) observed that regular participation in weight-bearing exercise significantly increases QUS parameters. However, the validity of QUS parameters for monitoring bone health in adolescents compared with DXA is not well known, and the studies using QUS in an active young population are scarce.

Recent studies have described controversial results correlating QUS and DXA in children and adolescents. Weeks et al (39) found not only positive and significant correlations between QUS and regional BMC and BMD DXA measurements but also disagreement between both techniques when children (4-18 y old) were ranked by age of peak height velocity quartiles. SI was positively correlated with total body BMD and BMC measured with DXA in children and adolescents (5-17 y old) (43). Similarly, BUA was significantly related to total body BMD in children (7-17 y old) (18). In contrast, a negative and significant correlation between SI and total body BMD was found in 4- to 8-year-old children, whereas no correlation was found with lumbar spine (LS) and leg BMD in this population (28). The age of the children may have influenced the results of these previous studies because it is known that woven bone tissue is often found in very young children and that woven bone is less stiff than other types of bone tissue (28). Also, a high degree of measurement error is observed in calcaneal QUS measures in very small or young children (39).

To the best of our knowledge, there are no studies investigating the relationship between DXA and QUS measures in active adolescents of a narrow age range. Therefore, the present study aims to investigate the correlations between DXA and QUS parameters and the intermethods agreement in 12- to 14-year-old active males.

\section{Methods}

\section{Subjects and Study Design}

The present investigation is a cross-sectional analysis as part of a 33-month longitudinal PRO-BONE study (effect of a program of short bouts of exercise on bone health in adolescents involved in different sports), of which the inclusion criteria and methods have been previously reported (37). The present investigation included 107 males aged 12-14 years at the beginning of the study, who comprised 3 cohorts: 41 swimmers, 37 footballers, and 29 cyclists. The inclusion and exclusion criteria were: 1) males aged 12-14 years engaged ( $\geq 3 \mathrm{~h} / \mathrm{wk}$ ) in osteogenic (football) and/or nonosteogenic (swimming and cycling) sports in the previous 3 years or more; 2) males aged 12-14 years not engaged in any of these sports ( $\geq 3 \mathrm{~h} / \mathrm{wk}$ ) in the last 3 or more years (control group); 3 ) participants not taking part in another clinical trial; 4) participants not having any acute infection lasting up to $<1$ week before inclusion; 5) participants had to be free of any medical history of diseases or medications affecting bone metabolism or the presence of an injury; and 6) white ethnicity.

Participants were recruited from athletic clubs across South West England. Written informed consent and assent forms were signed from parents and participants accordingly. The methods and procedures of the PRO-BONE study have been checked and approved by the Ethics Review Sector of Directorate-General of Research (European Commission, reference number: 618496), the Sport and Health Sciences Ethics Committee (University of Exeter, reference number: 2014/766), and the National Research Ethics Service Committee (NRES Committee South West-Cornwall and Plymouth, reference number: 14/SW/0060).

\section{Anthropometrics Measurements}

Stature (in centimeters) and body mass (in kilograms) were measured by using a stadiometer (Harpenden; Holtain Ltd, Crymych, UK; precision: $0.1 \mathrm{~cm}$; range: $60-210 \mathrm{~cm}$ ) and an electronic scale (Seca 877; Seca Ltd, Birmingham, UK; precision: $100 \mathrm{~g}$; range: 2-200 kg), respectively. Body mass index was calculated as body mass (in kilograms) divided by the height (in meters) squared. All anthropometric measurements were performed 3 times, and the means were calculated. Pubertal maturation was self-reported by the participants during each visit using adapted drawings of the 5 stages (Tanner) of pubertal hair development (30).

\section{Dual-Energy X-Ray Absorptiometry}

A DXA scanner (GE Lunar Healthcare Corp, Madison, WI) was used to measure BMC (in grams) for the LS (L1-L4), bilateral proximal femoral neck, and the total body. The total body scan was then used to obtain data for specific regions, such as legs and total body less head. The analyses were performed using GE enCORE software (2006, version 14.10.022; GE Healthcare, Madison, WI). The DXA equipment accuracy was checked on a daily basis before each scanning session, using the LS phantom as recommended by the manufacturer, and the same researcher performed all the scans. The positioning of the participants and the analyses of the results were undertaken according to the International Society for Clinical Densitometry (5). DXA have showed excellent precision for BMC (coefficient of variation $=0.6 \%$ ) in young athletes $(3)$.

\section{Qualitative Ultrasound}

QUS measurements were performed with a Lunar Achilles Insight (TM Insight; GE Healthcare, Milwaukee, WI) and the OsteoReport PC (software version 5.x+; GE Healthcare). The QUS uses ultrasound waves to measure the BUA and the SOS. The SI is then calculated by a linear combination of BUA and SOS as follows: $\mathrm{SI}=(0.67 \times \mathrm{BUA})+(0.28 \times \mathrm{SOS})-420$. The same device was used throughout the study, and the calibration was carried out prior to each visit, as in DXA. A standard procedure was followed 
according to the manufacturer's recommendations. All measurements were performed and analyzed by the same trained researcher. Participants were placed on a stable chair in a comfortable position directly in front of the Achilles device. The position of the leg rested lightly against the calf support, so the foot, calf, and thigh aligned with the center of the calf support and the positioner. Both feet were measured twice in the same session (right, left, right, left), and the mean was used for statistical analyses. The precision data for QUS in children have been reported as $0.2 \%$ for SOS, $1.5 \%$ for BUA, and $1.8 \%$ for stiffness (13).

\section{Physical Activity}

PA was objectively measured for 7 consecutive days, 24 hours per day using wrist accelerometers by using wrist accelerometers (GENEActiv; GENEA, Kimbolton, UK). Accelerometer is a sensor capable of measuring PA, providing objective measurements. The validity and reliability of the accelerometer have been established previously in children and adolescents (20). Participants were instructed to place the accelerometer on their nondominant wrist, and the time-sampling interval (epoch) was set at $100 \mathrm{~Hz}$ to obtain the data in 1-second epoch. For the present study, the time spent in moderate PA and vigorous PA was calculated using a cutoff point of 1140-3599 counts per minute and $\geq 3600$ counts per minute, respectively (20). Moderate to vigorous PA (MVPA) was calculated using a cutoff point of $\geq 1140$ counts per minute.

\section{Statistical Analysis}

Statistical analyses were performed using the SPSS IBM statistics (version 21.0 for Windows; SPSS Inc, Chicago, IL). Both statistical (Kolmogorov-Smirnov test) and visual checks (normal probability plots) were used to examine the normal distribution of the continuous variables. All the variables were normally distributed. Participants' characteristics were described as mean $(S D)$ and by quartiles of MVPA. MVPA was categorized as low (first quartile), average (second and third quartiles), and high (fourth quartile), and between group differences were obtained by analyses of variance.

Bivariate correlation coefficients were calculated to assess the raw relationships between DXA standard regions of interest and QUS parameters. Intraclass correlation coefficients (ICCs) with a 2-way mixed model (27) and Bland-Altman plots were used to assess the level of agreement between BMC regions derived from DXA and SI, using sample-specific $z$ score transformed data. Cutoffs of agreement in ICCs were defined by Fleiss (values of $>.75$ represent "excellent reliability" and values between .4 and .75 represent "fair to good reliability") (27).

Bland-Altman plots of the $z$ score transformed DXA and QUS outcomes were performed as a regression between the differences and averages of variables. When the spread of the differences increases with increasing mean, heteroscedasticity, using samplespecific $z$ score transformed data the limits of agreement for the differences are proportional to the mean (7).

Statistical analyses were performed with SPSS IBM software (v.19.0; SPSS Inc) except for Bland-Altman plot where Stata software (StataCorp LLC, Texas, USA) was used.

\section{Results}

Both statistical (Kolmogorov-Smirnov test) and graphical methods (normal probability plot) were used to examine the fit to a normal distribution for each variable. All fit acceptably to a normal distribution. Table 1 shows descriptive characteristics [mean $(S D)$ ] of the study sample. Participants with low MVPA score were older, taller, heavier, and had more lean mass and total body less head BMC than those with high MVPA score. Participants with average MVPA score were taller and heavier than those with high MVPA score.

Figure 1 shows bivariate correlations among DXA standard regions of interest BMC and QUS parameters. Overall, SI $(r=$ $.43-.52)$, BUA $(r=.50-.58)$, and SOS ( $r=.25-.27)$ were positively correlated with DXA-measured BMC, except SOS with the total body less head, legs, and LS. In general, DXA-measured BMC correlates better with BUA and SI than with SOS.

Table 2 shows ICCs among DXA-measured z-BMC and QUS-derived z-SI. Results show a fair to moderate intermethods agreement with ICCs ranging from .60 to .68.

The Bland-Altman analysis (Table 2) and plots (Figure 2) show mean differences between SI and DXA standard regions of interest BMC. A visual analysis of the plots of all the regions of interest also showed that there was a limited percentage of outliers $(3.2 \%-8.6 \%)$.

\section{Discussion}

The present study is, to our knowledge, the first to investigate agreement between DXA and QUS to assess bone health in active adolescent males. The main findings showed that most QUS parameters were positive and significantly correlated with DXA outcomes. Moreover, the present study found fair to good ICC of agreement between $z$ score transformed data from DXA and QUS to assess bone health in active adolescent males.

Although DXA is considered the gold standard and the most commonly used technique to assess bone status, QUS has been suggested as an efficient alternative method, specifically for largescale studies (18). Studies among adults have shown positive correlations between DXA and QUS parameters $(r=.20-.53)$ $(25,32)$. Also, QUS bone outcomes were significantly associated with risk of fractures in older women to a similar extent to DXA (15). Previous studies in children and adolescents indicated significantly positive correlations between DXA and QUS measurements at different skeletal sites $(4,18,43)$. This wide variability might be due to the use of different skeletal sites of measurement because some researchers use the radial site (4), whereas others use the calcaneal site $(18,43)$ as QUS parameters. In accordance with these previous studies, our data show a wide variability of correlations $(r=.15-.58)$, depending on the region and the QUS parameter assessed. Hip BMC demonstrated the strongest correlation with QUS, probably due to the trabecular-rich nature of the hip region (26) as well as calcaneus. In addition and similar to Rawal et al (23) and Wang et al (38), our data showed that SOS was poorly correlated with DXA measurements $(r=.15)$, in part perhaps because SOS is a volumetric parameter, while DXA variables are areal parameters (14). In any case, the fact that significant associations between 2 measures of the same parameter were observed does not imply concordance or does not prevent from clinical misclassification.

SI is the parameter used from the QUS manufacturer for the demographic comparison of patients. Moreover, the SI is the linear combination of BUA and SOS and is considered a better indicator of bone quality than BUA and SOS alone (43). Thus, we used $z$ scores of the SI to find the agreement between DXA and QUS parameters. Previous reports have shown controversial results 
Table 1 Descriptive Characteristics of the Participants

\begin{tabular}{|c|c|c|c|c|}
\hline & \multirow{2}{*}{$\frac{\text { Active adolescents }}{\text { All }}$} & \multicolumn{3}{|c|}{ MVPA scores } \\
\hline & & Low & Average & High \\
\hline$n$ & 107 & 26 & 53 & 26 \\
\hline \multicolumn{5}{|l|}{ Anthropometric data } \\
\hline age, y & $13.2(1.0)$ & $13.6(0.9)^{*}$ & $13.2(1.0)$ & $12.9(0.8)$ \\
\hline height, $\mathrm{cm}$ & $160.6(10.5)$ & $165.2(10.0)^{* *}$ & $161.6(10.4)^{*}$ & $154.1(8.3)$ \\
\hline body mass, $\mathrm{kg}$ & $48.8(10.1)$ & $53.8(11.2)^{* *}$ & $49.1(9.9)^{*}$ & $43.3(6.8)$ \\
\hline BMI, $\mathrm{kg} / \mathrm{m}^{2}$ & $18.7(2.2)$ & $19.5(2.5)$ & $18.6(2.2)$ & $18.1(1.5)$ \\
\hline \multicolumn{5}{|l|}{ pubertal maturation, $\%$} \\
\hline I & 18 & 2 & 9 & 8 \\
\hline II & 29 & 4 & 16 & 10 \\
\hline III & 21 & 7 & 11 & 4 \\
\hline IV & 30 & 11 & 16 & 4 \\
\hline $\mathrm{V}$ & 2 & 1 & 1 & 0 \\
\hline MVPA, min/d & $103.7(34.0)$ & & & \\
\hline lean mass, kg & $38.4(8.4)$ & $42.5(8.8)^{*}$ & $38.3(8.5)$ & $34.2(6.0)$ \\
\hline percentage of body fat, $\%$ & $15.8(6.8)$ & $18.9(8.3)$ & $19.5(7.4)$ & $18.9(5.0)$ \\
\hline \multicolumn{5}{|l|}{ QUS measurements } \\
\hline $\mathrm{SOS}, \mathrm{m} / \mathrm{s}$ & $1586.64(23.21)$ & $1585.62(26.62)$ & $1584.88(25.16)$ & $1591.31(19.49)$ \\
\hline BUA, $\mathrm{mB} / \mathrm{mHz}$ & $104.36(11.54)$ & $103.61(10.60)$ & $105.4(12.20)$ & $103.4(11.63)$ \\
\hline SI & 94.09 (11.99) & $93.27(10.88)$ & $94.30(13.14)$ & $94.76(11.36)$ \\
\hline \multicolumn{5}{|l|}{ DXA measurements, $\mathrm{g}$} \\
\hline TBLH BMC & $1540.43(347.50)$ & $1666.95(343.62)^{*}$ & 1558.13 & $1388.46(311.17)$ \\
\hline legs BMC & $759.14(163.43)$ & 799.23 (155.57) & 774.94 (164.24) & $694.24(159.18)$ \\
\hline hip BMC & $28.49(5.85)$ & $29.67(5.53)$ & $28.75(6.09)$ & $27.00(5.7)$ \\
\hline FN BMC & $4.47(0.72)$ & $4.57(0.76)$ & $4.49(0.72)$ & $4.36(0.69)$ \\
\hline LS BMC & $40.61(10.55)$ & $44.04(11.00)$ & $40.69(11.10)$ & $37.06(8.21)$ \\
\hline
\end{tabular}

Note. Data are presented as mean $(S D)$.

Abbreviations: BMC, bone mineral content; BMI, body mass index; BUA, broadband ultrasound attenuation; DXA, dualenergy X-ray absorptiometry; FN, femoral neck; LS, lumbar spine; MVPA, moderate to vigorous physical activity; QUS, quantitative ultrasound; SI, stiffness index; SOS, speed of sound; TBLH, total body less head.

*Significant difference with high MVPA score $(P<.05)$. * Significant difference with high MVPA score $(P<.001)$.

Table 2 ICC and Bland-Altman Analysis Between DXA Standard Regions of Interest BMC and QUS (SI)

\begin{tabular}{llccc}
\hline & \multicolumn{2}{c}{$\begin{array}{c}\text { ICC }_{\mathbf{3 , 1}} \text { DXA- } \\
\text { QUS }\end{array}$} & & Bland-Altman analysis \\
\cline { 2 - 3 } \cline { 5 - 5 } & $\boldsymbol{r}$ & $\mathbf{9 5 \%} \mathbf{~ C l}$ & & $\mathbf{9 5 \% ~ L O A ~}$ \\
\hline TBLH BMC & .61 & $.43-.73$ & & -2.112 to 2.112 \\
Legs BMC & .61 & $.43-.74$ & & -2.103 to 2.103 \\
Hip BMC & .68 & $.54-.78$ & & -1.952 to 1.952 \\
FN BMC & .68 & $.53-.58$ & & -1.966 to 1.966 \\
LS BMC & .60 & $.41-.73$ & & -2.126 to 2.126 \\
\hline
\end{tabular}

Note. $N=107$. Data are $z$ score transformed.

Abbreviations: BMC, bone mineral content; CI, confidence interval; DXA, dualenergy X-ray absorptiometry (LUNAR); FN, femoral neck; $\mathrm{ICC}_{3,1}, 2$-way mixed single measures model of intraclass correlation coefficient; LOA, limits of agreement; LS, lumbar spine; QUS, quantitative ultrasound; SI, stiffness index; TBLH, total body less head.

regarding the agreement of DXA and calcaneal QUS parameters. Previously, Kappa analysis showed that the diagnostic agreement between both methods in spine and femur sites was generally poor in adults (10), although calcaneal QUS can assess hip fracture patients equally as well as DXA hip assessment in elderly women (12). In children, calcaneal BUA has demonstrated poor agreement with absolute BMC and BMD derived from DXA (39), and poor agreement of radial SOS with whole-body BMD (4). The present study found fair to good ICC between the 2 techniques using $z$ scores despite the fact that $3.2 \%-8.6 \%$ of the participants fell outside the LOAs. Previous studies found similar results in predicting the long-term risk of fractures among elderly people (17). It is known that the disagreement between measurements may exist in different skeletal sites due to the measurements performed using different techniques and devices. These discrepancies could reflect the true anatomic variation related to genetic, environmental, or physiological factors (9). To our knowledge, there are no studies analyzing the agreement of both tools in athletic adolescents, and it is well known that sport activities could have an osteogenic function in a growing skeleton. Moreover, this study is novel in the examination of DXA and QUS parameters in adolescents of a narrow age range, as most studies in youth demonstrated relationships between both devices in samples of broad age ranges.

The current study has several limitations that should be acknowledged. First, the cross-sectional design of our study prevents us from making cause-effect inferences. Second, we 

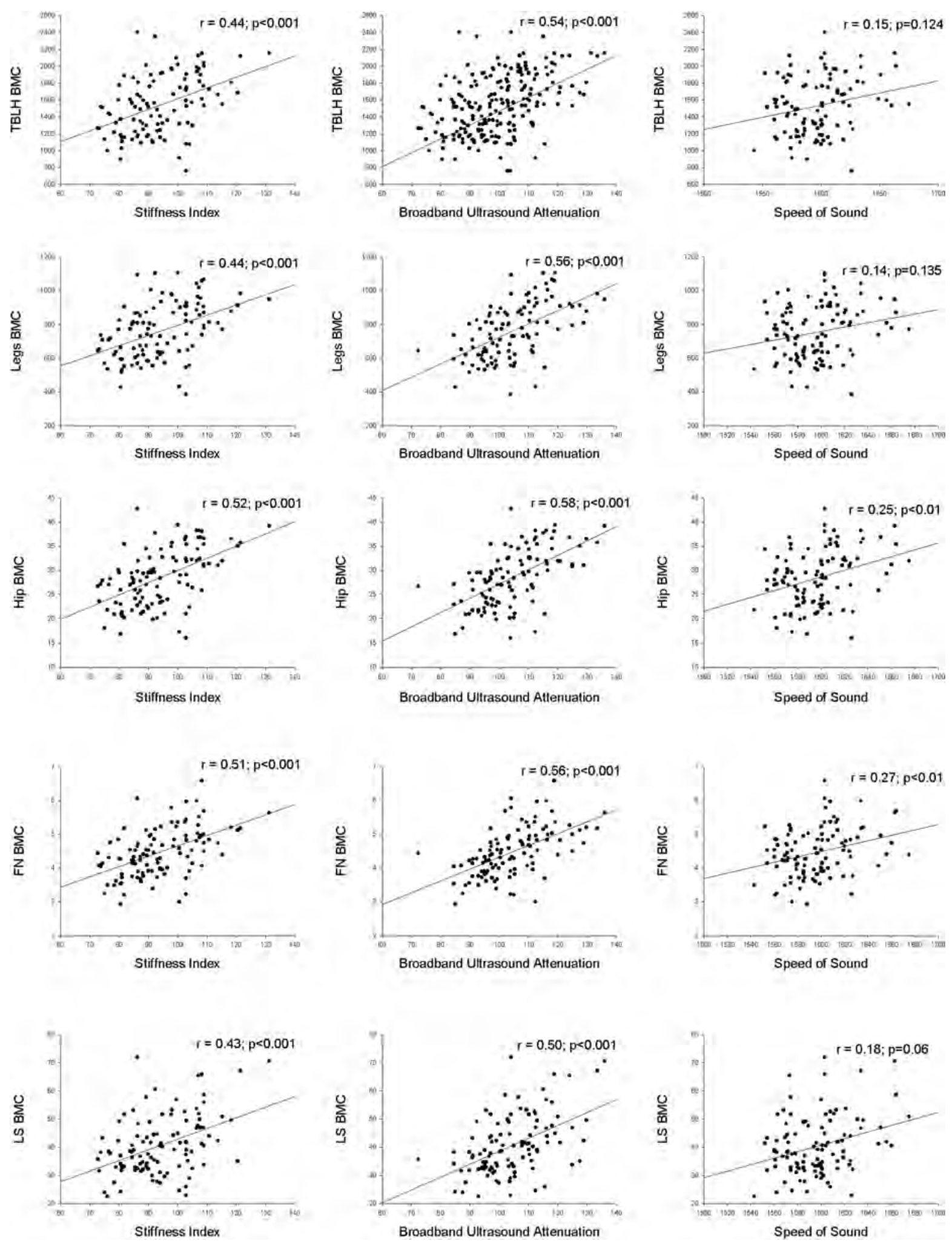

Figure 1 - Bivariate correlation coefficients between DXA standard regions of interest BMC $(\mathrm{g})$ and QUS parameters $(\mathrm{SOS}, \mathrm{m} / \mathrm{s} ; \mathrm{BUA}, \mathrm{mB} / \mathrm{mHz})$ in active adolescents. BMC indicates bone mineral content; BUA, broadband ultrasound attenuation; DXA, dual-energy X-ray absorptiometry; FN, femoral neck; LS, lumbar spine; QUS, quantitative ultrasound; SI, stiffness index; SOS, speed of sound; TBLH, total body less head. 

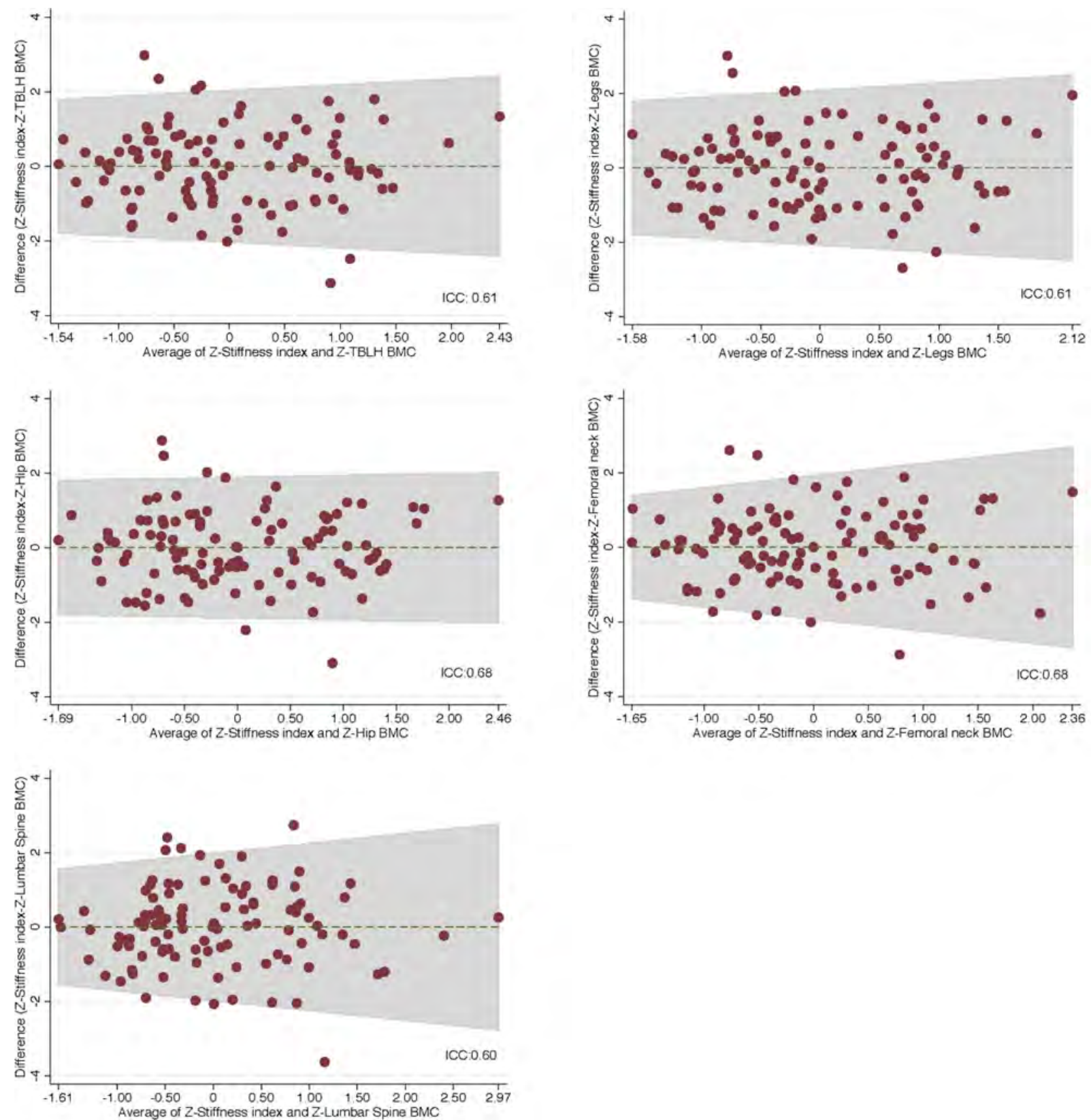

Figure 2 - Bland-Altman plot showing the limits of agreement between stiffness index $z$ score and BMC $z$ score. The shading on the Bland-Altman plot represents the heteroscedasticity taking into account the magnitude of the differences. The central line represents the intermethods difference (bias), which is 0 as $z$ scores have been used. BMC indicates bone mineral content; SI, stiffness index; TBLH, total body less head; ICC, intraclass correlation coefficient; LS, lumbar spine; FN, femoral neck.

did not examine the foot dimensions, and it has been reported that foot length is an independent predictor of the QUS parameter in males (33). Third, considering the small sample size of our study, the adjustment by type of sport and Tanner status was not possible. Fourth, accelerometers only register accelerations, and therefore, we may have underestimated the intensity of activities with extra weight as well as those that relate to nonweight-bearing efforts. Fifth, as many different types of DXA and QUS scanners are available, our results may not be generalizable across any devices than the GE Lunar and Lunar Achilles Insight. Finally, our analyses were not a site-to-site comparison between the 2 methods, as different sites are made of varying proportions of trabecular and cortical bone and experience different loading patterns. Nevertheless, it should be noted that further studies are needed to assess similar regions (calcaneal by QUS and DXA) to make comparisons between both techniques.

\section{Conclusion}

Currently, DXA is the reference standard for assessing bone health in children and adolescents. However, $z$ scores of DXA-derived $\mathrm{BMC}$ and calcaneal SI showed not only moderate and positive 
correlations but also fair to good agreement and no symptoms of heteroscedasticity in the Bland-Altman analysis. Therefore, the QUS could represent an acceptable method for assessing bone outcomes in active adolescent males.

\section{Acknowledgments}

The authors gratefully acknowledge the sport coaches, schoolteachers, and the Children's Health and Exercise Research Centre (CHERC) research team for their help in running the study.

\section{References}

1. Baroncelli GI. Quantitative ultrasound methods to assess bone mineral status in children: technical characteristics, performance, and clinical application. Pediatr Res. 2008;63(3):220-8. doi:10.1203/ PDR.0b013e318163a286

2. Baxter-Jones ADG, Faulkner RA, Forwood MR, Mirwald RL, Bailey DA. Bone mineral accrual from 8 to 30 years of age: an estimation of peak bone mass. J Bone Miner Res. 2011;26(8):1729-39. PubMed doi:10.1002/jbmr.412

3. Bilsborough JC, Greenway K, Opar D, Livingstone S, Cordy J, Coutts AJ. The accuracy and precision of DXA for assessing body composition in team sport athletes. J Sports Sci. 2014;32(19):1821-8. PubMed doi:10.1080/02640414.2014.926380

4. Chong KH, Poh BK, Jamil NA, Kamaruddin NA, Deurenberg P. Radial quantitative ultrasound and dual energy x-ray absorptiometry: intermethod agreement for bone status assessment in children. BioMed Res Int. 2015;2015:232876. PubMed

5. Crabtree NJ, Arabi A, Bachrach LK, et al. Dual-energy X-ray absorptiometry interpretation and reporting in children and adolescents: the revised 2013 ISCD pediatric official positions. J Clin Densitom. 2014;17(2):225-42. PubMed doi:10.1016/j.jocd.2014.01.003

6. Davies JH, Evans BA, Gregory JW. Bone mass acquisition in healthy children. Arch Dis Child. 2005;90(4):373-8. PubMed doi:10.1136/ adc. 2004.053553

7. Euser AM, Dekker FW, le Cessie S. A practical approach to BlandAltman plots and variation coefficients for log transformed variables. J Clin Epidemiol. 2008;61(10):978-82. PubMed doi:10.1016/ j.jclinepi.2007.11.003

8. Gracia-Marco L, Moreno LA, Ortega FB, et al. Levels of physical activity that predict optimal bone mass in adolescents: the HELENA study. Am J Prev Med. 2011;40(6):599-607. PubMed doi:10.1016/ j.amepre.2011.03.001

9. Grampp S, Henk C, Lu Y, et al. Quantitative US of the calcaneus: cutoff levels for the distinction of healthy and osteoporotic individuals. Radiology. 2001;220(2):400-5. PubMed doi:10.1148/ radiology.220.2.r01au05400

10. Grampp S, Henk CB, Fuerst TP, et al. Diagnostic agreement of quantitative sonography of the calcaneus with dual X-ray absorptiometry of the spine and femur. Am J Roentgenol. 1999;173(2): 329-34. doi:10.2214/ajr.173.2.10430129

11. Hadji P, Hars O, Wuster C, et al. Stiffness index identifies patients with osteoporotic fractures better than ultrasound velocity or attenuation alone. Maturitas. 1999;31(3):221-6. PubMed doi:10.1016/ S0378-5122(99)00003-1

12. He YQ, Fan B, Hans D, et al. Assessment of a new quantitative ultrasound calcaneus measurement: precision and discrimination of hip fractures in elderly women compared with dual X-ray absorptiometry. Osteoporos Int. 2000;11(4):354-60. PubMed doi:10.1007/ s001980070125
13. Jaworski M, Lebiedowski M, Lorenc RS, Trempe J. Ultrasound bone measurement in pediatric subjects. Calcif Tissue Int. 1995;56(5): 368-71. PubMed doi:10.1007/BF00301604

14. Langton CM, Langton DK. Comparison of bone mineral density and quantitative ultrasound of the calcaneus: site-matched correlation and discrimination of axial BMD status. Br J Radiol. 2000;73(865):31-5. doi:10.1259/bjr.73.865.10721317

15. Marin F, Gonzalez-Macias J, Diez-Perez A, Palma S, DelgadoRodriguez M. Relationship between bone quantitative ultrasound and fractures: a meta-analysis. J Bone Miner Res. 2006;21(7): 1126-35. PubMed doi:10.1359/jbmr.060417

16. Moayyeri A, Adams JE, Adler RA, Krieg MA, Hans D, Compston J, Lewiecki EM. Quantitative ultrasound of the heel and fracture risk assessment: an updated meta-analysis. Osteoporos Int. 2012; 23(1):143-53. PubMed doi:10.1007/s00198-011-1817-5

17. Moayyeri A, Kaptoge S, Dalzell N, et al. Is QUS or DXA better for predicting the 10-year absolute risk of fracture? J Bone Miner Res. 2009;24(7):1319-25. PubMed doi:10.1359/jbmr.090212

18. Mughal MZ, Langton CM, Utretch G, Morrison J, Specker BL. Comparison between broad-band ultrasound attenuation of the calcaneum and total body bone mineral density in children. Acta Paediatr. 1996;85(6):663-5. PubMed doi:10.1111/j.1651-2227.1996. tb14119.x

19. Njeh CF, Fuerst T, Hans D, Blake GM, Genant HK. Radiation exposure in bone mineral density assessment. Appl Radiat Isot. 1999; 50(1):215-36. PubMed doi:10.1016/S0969-8043(98)00026-8

20. Phillips LR, Parfitt G, Rowlands AV. Calibration of the GENEA accelerometer for assessment of physical activity intensity in children. J Sci Med Sport. 2013;16(2):124-8. PubMed doi:10.1016/j.jsams. 2012.05.013

21. Prins SH, Jorgensen HL, Jorgensen LV, Hassager C. The role of quantitative ultrasound in the assessment of bone: a review. Clin Physiol. 1998;18(1):3-17. PubMed doi:10.1046/j.1365-2281.1998. 00067.x

22. Raum K, Grimal Q, Varga P, Barkmann R, Gluer CC, Laugier P. Ultrasound to assess bone quality. Curr Osteoporos Rep. 2014;12(2): 154-62. PubMed doi:10.1007/s11914-014-0205-4

23. Rawal J, Eleftheriou K, Skipworth J, et al. Relationship between calcaneal quantitative ultrasound and hip dual energy X-ray absorptiometry in young healthy men. Osteoporos Int. 2012;23(7):1947-56. PubMed doi:10.1007/s00198-011-1853-1

24. Rizzoli R, Bianchi ML, Garabédian M, McKay HA, Moreno LA. Maximizing bone mineral mass gain during growth for the prevention of fractures in the adolescents and the elderly. Bone. 2010;46(2): 294-305. PubMed doi:10.1016/j.bone.2009.10.005

25. Sandstrom L, McGuigan FE, Callreus M, Akesson KE. Peak bone mass and quantitative ultrasound bone properties in young adulthood: a study in the PEAK-25 cohort of women. J Clin Densitom. 2016; 19(4):477-84. PubMed doi:10.1016/j.jocd.2016.03.001

26. Schott AM, Weill-Engerer S, Hans D, Duboeuf F, Delmas PD, Meunier PJ. Ultrasound discriminates patients with hip fracture equally well as dual energy X-ray absorptiometry and independently of bone mineral density. J Bone Miner Res. 1995;10(2):243-9. PubMed doi:10.1002/jbmr.5650100210

27. Shrout PE, Fleiss JL. Intraclass correlations: uses in assessing rater reliability. Psychol Bull. 1979;86(2):420-8. PubMed doi:10.1037/ 0033-2909.86.2.420

28. Sioen I, Goemare S, Ahrens W, et al. The relationship between paediatric calcaneal quantitative ultrasound measurements and dual energy X-ray absorptiometry (DXA) and DXA with laser (DXL) as well as body composition. Int J Obes. 2011;35 Suppl1:S125-30. doi:10.1038/ijo.2011.44 
29. Stewart A, Reid DM. Quantitative ultrasound in osteoporosis. Semin Musculoskelet Radiol. 2002;6(3):229-32. PubMed doi:10.1055/ s-2002-36720

30. Tanner JM, Whitehouse RH. Clinical longitudinal standards for height, weight, height velocity, weight velocity, and stages of puberty. Arch Dis child. 1976;51(3):170-9. PubMed doi:10.1136/ adc.51.3.170

31. Torres-Costoso A, Gracia-Marco L, Sanchez-Lopez M, NotarioPacheco B, Arias-Palencia N, Martinez-Vizcaino V. Physical activity and bone health in schoolchildren: the mediating role of fitness and body fat. PLoS ONE. 2015;10(4):e0123797. PubMed doi:10.1371/ journal.pone.0123797

32. Trimpou P, Bosaeus I, Bengtsson BA, Landin-Wilhelmsen K. High correlation between quantitative ultrasound and DXA during 7 years of follow-up. Eur J Radiol. 2010;73(2):360-4. doi:10.1016/j.ejrad .2008 .11 .024

33. van den Bergh JP, Noordam C, Ozyilmaz A, Hermus AR, Smals AG, Otten BJ. Calcaneal ultrasound imaging in healthy children and adolescents: relation of the ultrasound parameters BUA and SOS to age, body weight, height, foot dimensions and pubertal stage. Osteoporos Int. 2000;11(11):967-76. PubMed doi:10.1007/ s001980070036

34. Vicente-Rodríguez G. How does exercise affect bone development during growth? Sports Med. 2006;36(7):561-9. doi:10.2165/ 00007256-200636070-00002

35. Vicente-Rodriguez G, Ara I, Perez-Gomez J, Serrano-Sanchez JA, Dorado C, Calbet JA. High femoral bone mineral density accretion in prepubertal soccer players. Med Sci Sports Exerc. 2004;36(10): 1789-95. PubMed doi:10.1249/01.MSS.0000142311.75866.D7

36. Vlachopoulos D, Barker AR, Ubago-Guisado E, et al. The effect of 12-month participation in osteogenic and non-osteogenic sports on bone development in adolescent male athletes. The PRO-BONE study. J Sci Med Sport. 2018;21(4):404-409. PubMed doi:10. 1016/j.jsams.2017.08.018
37. Vlachopoulos D, Barker AR, Williams CA, Knapp KM, Metcalf BS, Gracia-Marco L. Effect of a program of short bouts of exercise on bone health in adolescents involved in different sports: the PRO-BONE study protocol. BMC Public Health. 2015;15:361. PubMed doi:10.1186/s12889-015-1633-5

38. Wang Q, Nicholson PH, Timonen J, Alen M, Moilanen P, Suominen $\mathrm{H}$, Cheng S. Monitoring bone growth using quantitative ultrasound in comparison with DXA and pQCT. J Clin Densitom. 2008;11(2): 295-301. PubMed doi:10.1016/j.jocd.2007.10.003

39. Weeks BK, Hirsch R, Nogueira RC, Beck BR. Is calcaneal broadband ultrasound attenuation a valid index of dual-energy x-ray absorptiometry-derived bone mass in children? Bone Joint Res. 2016;5(11): 538-43. PubMed doi:10.1302/2046-3758.511.BJR-2016-0116.R1

40. Wetter AC, Economos CD. Relationship between quantitative ultrasound, anthropometry and sports participation in college aged adults. Osteoporos Int. 2004;15(10):799-806. PubMed doi:10.1007/s00198004-1607-4

41. Wilkinson K, Vlachopoulos D, Klentrou P, et al. Soft tissues, areal bone mineral density and hip geometry estimates in active young boys: the PRO-BONE study. Eur J Appl Physiol. 2017;117(4):83342. PubMed doi:10.1007/s00421-017-3568-2

42. Willson T, Nelson SD, Newbold J, Nelson RE, LaFleur J. The clinical epidemiology of male osteoporosis: a review of the recent literature. Clin Epidemiol. 2015;7:65-76. PubMed

43. Xu Y, Guo B, Gong J, Xu H, Bai Z. The correlation between calcaneus stiffness index calculated by QUS and total body BMD assessed by DXA in Chinese children and adolescents. J Bone Miner Metab. 2014;32(2):159-66. PubMed doi:10.1007/s00774013-0474-5

44. Yung PS, Lai YM, Tung PY, Tsui HT, Wong CK, Hung VW, Qin L. Effects of weight bearing and non-weight bearing exercises on bone properties using calcaneal quantitative ultrasound. Br J Sports Med. 2005;39(8):547-51. PubMed doi:10.1136/bjsm.2004.014621 